\title{
In defence of the self-location uncertainty account of probability in the many-worlds interpretation
}

\author{
Kelvin J. McQueen* and Lev Vaidman ${ }^{\dagger}$
}

February 13, 2018

\begin{abstract}
We defend the many-worlds interpretation of quantum mechanics (MWI) against the objection that it cannot explain why measurement outcomes are predicted by the Born probability rule. We understand quantum probabilities in terms of an observer's self-location probabilities. We formulate a probability postulate for the MWI: the probability of selflocation in a world with a given set of outcomes is the absolute square of that world's amplitude. We provide a proof of this postulate, which assumes the quantum formalism and two principles concerning symmetry and locality. We also show how a structurally similar proof of the Born rule is available for collapse theories. We conclude by comparing our account to the recent account offered by Sebens and Carroll.
\end{abstract}

\section{Introduction}

Quantum theory is more than a century old and is arguably our most successful physical theory. There is a consensus that both its deterministic and its statistical predictions are fully satisfactory. However, there is still no consensus about the correct interpretation. The main difficulty is the measurement problem: after a measurement, the basic formalism (i.e. the wavefunction evolving according to the Schrödinger equation) corresponds to a superposition of all possible outcomes, but it seems that we observe just one of these outcomes.

In our view, the best interpretation is the many-worlds interpretation (MWI) [Everett, 1957]. According to the MWI, a measurement device in a superposition of depicting all possible outcomes is understood as a multiplicity of measuring devices, each depicting a single outcome in a different world, and so it only seems like there is one outcome.

Currently, the MWI is far from being the leading interpretation of quantum mechanics and apparently the reason for this is an extensive criticism of its ability to deal with the issue of probability. In this paper we will argue that the MWI competes well with other interpretations regarding this issue.

The future-directed question: What is the probability of obtaining a particular result in a quantum measurement that will be performed? is more difficult to deal with in the framework of the MWI than in other interpretations. We will confront this question in Section 3, with help from the idea of self-location uncertainty. Before this, in Section 2, we will discuss how

* Department of Philosophy, Chapman University, CA 92866, United States.

$\dagger$ Raymond and Beverly Sackler School of Physics and Astronomy, Tel-Aviv University, Tel-Aviv 69978, Israel. 
various interpretations explain the statistics of quantum experiments which have been performed. Section 4.1 offers a proof of the orthodox probability rule (the Born rule) in collapse theories. Section 4.2 then provides a structurally similar proof of the analogue probability rule - what we call the probability postulate - in the MWI. Section 4.3 explains how the MWI is confirmed, despite its prediction of worlds with maverick experimental statistics. Finally, in section 5 we strengthen Kent's [2015] objection to the self-location uncertainty account of Sebens and Carroll [forthcoming]. We show that Sebens and Carroll's proof cannot be modified to avoid Kent's objection.

\section{Statistics of the results of quantum experiments}

Quantum theory makes both deterministic and statistical predictions. A typical example is a Stern-Gerlach experiment which measures a spin- $\frac{1}{2}$ particle. The Schrödinger equation explains the result of measuring the spin in the $z$ direction given that it was prepared in an eigenstate of $z$-spin. But when the spin is prepared in a superposition of eigenstates of $z$-spin, the Schrödinger equation tells us that the measuring device and the observer will be in a superposition of states corresponding to the two possible outcomes. Moreover, by repeating quantum experiments we obtain statistical results which the Schrödinger equation does not seem to explain, so apparently we need to add something. Let us review the explanations of the observed statistics of quantum experiments provided by various interpretations of quantum mechanics.

The textbook solution postulates that the wavefunction collapses to an eigenstate of the measured variable at the time of measurement. The probability of collapse to a particular eigenstate is the absolute square of the amplitude of the eigenstate in the superposition; this is the Born rule, which governs the statistics of measurement outcomes.

Physical collapse theories ([Pearle, 1976], [Ghirardi, Rimini, \& Weber, 1986]) postulate genuinely random localization processes happening with probability equal to the absolute square of the amplitude of the wavefunction of every particle, causing a collapse of the wavefunction of macroscopic objects, like detectors, in measurement-type situations. This leads to Born rule statistics.

Bohmian mechanics [Bohm, 1952] solves the measurement problem without postulating wavefunction collapse. It introduces additional elements: Bohmian positions for all particles. It postulates that all macroscopic objects supervene on these additional elements. The role of the wavefunction is to govern the evolution of Bohmian particles and thus the evolution of objects. Whereas the wavefunction has parts corresponding to all possible outcomes, the set of Bohmian particle positions corresponds to a single outcome. The observed statistics of quantum measurements are obtained from the distribution of the Bohmian particle positions at the beginning of the universe. The postulate about this distribution leads to Born rule statistics.

In all the above theories there is some postulate in addition to the Schrödinger equation, which ensures that the outcomes of quantum experiments exhibit Born rule statistics.

In the MWI there is nothing that leads to a single outcome of a quantum experiment. There is no collapse of the wavefunction to a single branch with a specific outcome, and there is no additional entity that singles out one branch as the "real" outcome. The MWI states that a single outcome is an illusion. All possible outcomes take place. The observers do not experience multiple outcomes because they are constituted this way. There are multiple outcomes, but also multiple observers. The MWI, or at least the MWI that we advocate, has an additional postulate (Vaidman [2014: 4.2]):

MWI probability postulate: The probability of self-location in a world with a given set of outcomes is the absolute square of that world's amplitude. 
This postulate is needed to explain observed frequencies of outcomes of quantum experiments. It has operational meaning for an observer who has records of setups of performed quantum experiments, but is not aware of their results. Based on these records he can calculate what the possible outcomes are and what the coefficients in the quantum state corresponding to those outcomes are. The absolute square of a given coefficient represents the probability of self-location in a world whose results correspond to that coefficient.

In contrast to collapse theories, the postulate is not about some fundamental physical process, it is about the experience of the observer. Each observer with records of some quantum experiments understands that there are many other observers living in parallel worlds who obtained different outcomes. The postulate concerns the subjective probability for an observer to live in a particular world.

This explanation leaves a number of questions about our postulate unanswered. What are the exact specifications of situations involving self-location uncertainty? Why should self-location probabilities correspond to the Born rule and not some other rule? And how can the postulate make sense in light of the existence of maverick worlds, worlds in which observers obtain low probability results? We answer these questions in the following sections.

\section{The idea of quantum self-location uncertainty}

Consider a quantum experiment with two possible results, $a$ and $b$. Before performing the experiment, the experimenter might ask herself: What is the probability of obtaining a particular result? It is difficult to make sense of this question in the MWI. In contrast to collapse theories, there is no chance. So the probability of obtaining a particular result cannot be the chance that nature will select that result. And in contrast to Bohmian mechanics, there is no ignorance about the physical state. So the probability of a result cannot be the likelihood of the result in light of limited knowledge about the system. Given the MWI, the experimenter knows that the measurement will create two descendants, one that obtains result $a$, another that obtains $b$. There is no uncertainty as to which descendant the experimenter will become, for she does not become any one person. This difficulty, of making sense of probabilistic statements in the MWI, is referred to as the incoherence problem.

But now imagine that the experimenter performs the experiment without looking at the result; she instead arranges to be put to sleep with a sleeping pill and taken to room $A$ if the result was $a$, and room $B$ if the result was $b$. The rooms are identical from the inside. So when each of the experimenter's descendants wake up, they will be uncertain as to which room they are in, and therefore uncertain as to which result, $a$ or $b$, obtains in their own world. The question: What is the probability for result $a$ ? makes sense for them. It is not a question about what happened, it is a question about their self-location. The descendants might know everything relevant regarding the wavefunction of the universe, but still be ignorant about who they are. The descendants are in states of self-location uncertainty.

This solution to the incoherence problem, originally proposed in Vaidman [1998: sec. 8], uses the uncertainty of the observers at the time after the measurement was performed but before they become aware of their respective results. All descendants will ascribe the same probabilities because they all descended from the same experimenter, and therefore inherit identical dispositions from that experimenter. Unlike her descendants, the pre-measurement experimenter will not assign probabilities to the results, since she is certain that all results will be obtained. But if she believes the MWI, then we can make sense of why her betting behaviour would still be equivalent to the betting behaviour of an agent who believes that only one result is obtained. If her descendants assign probability $p>0.5$ to being in the $a$-world, then her descendants will 
want her to have placed a bet whose maximum payoff is received in the $a$-world. And since it is the descendants that reap the rewards, it is rational for her to satisfy the wants of her descendants and bet as if result $a$ will happen with probability $p$.

The MWI ontology offers an additional resource for rationalizing the behaviour of premeasurement experimenters who believe the MWI. It offers a sense in which some descendants have a greater measure of existence [Vaidman, 1998; Greaves, 2004; Groisman et. al., 2013] than other descendants, in a way which may make pre-measurement agents care more about descendants that exist more.

It is important to emphasise that the pre-measurement experimenter will apply such a caring measure in the absence of any uncertainty about the future. This subtlety has not been appreciated by Albert [2015: p177] who objects that the uncertainty of the pre-observation descendants "comes too late in the game". Albert is trying to infer pre-measurement uncertainty from postmeasurement pre-observation uncertainty. He assumes that "if there is something about the world of which these descendants are uncertain, the premeasurement observer must have been uncertain about that too!"

Lewis' objection [2007: p5; 2016: p131] misses the mark for similar reasons. Lewis argues that "after-the-fact probabilities are not predictive in any obvious sense; the observer may be uncertain about what has happened, but not about what will happen. That is, the uncertainty seems to be in the wrong place to play any role in decision." Lewis then complains that "it is not clear what these numbers represent, since there is no pre-measurement uncertainty." But as emphasised, these numbers represent an aspect of the agent's care for her descendants, and are therefore predictive of behaviour. They predict that the pre-measurement experimenter will place bets that favour worlds that are more probable from her pre-observation descendant's perspective.

Let us now specify more carefully what it takes for a descendant to experience genuine selflocation uncertainty. To do this we will analyze a few variations of a familiar case. You are a physics professor who instructs a student to perform particular Stern-Gerlach experiments on a spin- $\frac{1}{2}$ particle prepared in a specific state. You also instruct the student to write the results in a notebook and then bring it to you. Prima facie before you open the notebook you are in a position of self-location uncertainty - you do not know what world you are in. Once you open the notebook you will discover that you are in a world with a specific result. But there is more to this example than meets the eye. We discuss three possible cases, which illustrate what we call absent, tainted, and clean, self-location uncertainty.

Absent self-location uncertainty. The student performs the measurement and enters into a superposition of writing " $\uparrow$ " and writing " $\downarrow$ ", in the notebook. The student places the notebook on your table while you are away, exactly parallel to the edge of the table, and then leaves. Later, you return and see the unopened notebook. Are you in a position of self-location uncertainty? It is feasible that you do not become entangled with the records inside the notebook at this stage. In that case the quantum description is:

$$
\left.\left.|\psi\rangle_{\text {absent }}=\frac{1}{\sqrt{3}}|\uparrow\rangle_{p}|\square, \uparrow\rangle_{n} \mid \text { see }: \square\right\rangle_{y}+\sqrt{\frac{2}{3}}|\downarrow\rangle_{p}|\square, \downarrow\rangle_{n} \mid \text { see }: \square\right\rangle_{y} .
$$

Here $|\square, \uparrow\rangle_{n}$ is the state of the notebook in which it is positioned parallel to the edge of the table,

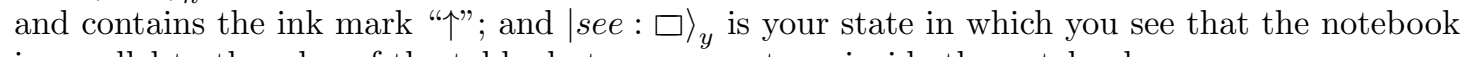
is parallel to the edge of the table, but you cannot see inside the notebook.

Although the internal state of the notebook, $n$, is entangled with the particle, $p$, it might well not be entangled with your body. But then you, $y$, have not branched, and may not branch until you open it. Consequently, when you see it on your desk and ask, "what is the probability that 
I am in the world with the spin- $\uparrow$ result?", you ask a meaningless question. For prior to having branched, there is only one "you" that is in both the world with the spin- $\uparrow$ result and the world with the spin- $\downarrow$ result. Self-location uncertainty is therefore absent.

Why are there two worlds? One condition we impose on what it takes to be a world is that every macroscopic object in a world must be well-localised. Equation (1) describes two macroscopically different states of a notebook (records of spin- $\uparrow$ and spin- $\downarrow$ ), so there are two worlds. It shows that the same state of the professor belongs to both worlds.

We can imagine different definitions of "world". One might follow Everett [1957] and stipulate that worlds are relative to the observer and they branch only when the observer branches. In Everett's language (1) would involve one world relative to you (and in virtue of there being only one world, there would clearly be no self-location uncertainty for Everett too).

Tainted self-location uncertainty. The student again enters into a superposition of writing

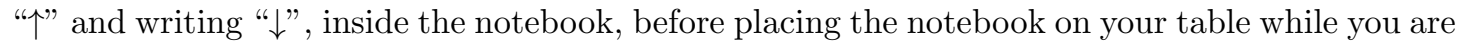
away. But this time, he places the notebook on the table with a different orientation, depending on the result of the measurement. Later, you return and see the unopened notebook. Are you in a position of self-location uncertainty? The quantum description is:

$$
\left.\left.|\psi\rangle_{\text {tainted }}=\frac{1}{\sqrt{3}}|\uparrow\rangle_{p}|\square, \uparrow\rangle_{n} \mid \text { see }: \square\right\rangle_{y}+\sqrt{\frac{2}{3}}|\downarrow\rangle_{p}|\triangleleft, \downarrow\rangle_{n} \mid \text { see }: \diamond\right\rangle_{y} .
$$

It is clear from (2) that there are two worlds specified by the orientation of the notebook: "parallel" and "rotated" and each of your descendants is aware in which world he is located. The spin is $\uparrow$ for the descendant who sees the notebook parallel to the edge of the table, and $\downarrow$ for the descendant who sees the rotated notebook. However, your descendants don't know the correlation, so they don't know what is in their respective notebooks. Each descendant does not know if he is located in a world with spin $\uparrow$ or in a world with spin $\downarrow$. This type of self-location uncertainty is tainted in the following sense: if they knew the quantum state, then they would not be in a position of self-location uncertainty. The source of the uncertainty is the descendant's ignorance of certain physical facts.

Note that despite the uncertainty being tainted, the probability postulate is still applicable to this situation. The absolute square of the amplitude of the spin- $\uparrow$ component is the probability for a descendant to be located in the spin- $\uparrow$ world.

Clean self-location uncertainty. Clean self-location uncertainty is not easy to come by. We need that the professor's brain be split into different worlds, but that his perception in different worlds does not distinguish between those worlds. A gedankenexperiment involves the student placing the notebook in the same way, but gently rotating the entire building which contains the professor in the case of spin- $\downarrow$. The professor, who returns to the room and sees the notebook, has branched. The quantum state is:

$$
\left.|\psi\rangle_{\text {clean }}=\frac{1}{\sqrt{3}}|\uparrow\rangle_{p}|\square, \uparrow\rangle_{n} \mid \text { see }: \square\right\rangle_{y}+\sqrt{\frac{2}{3}}|\downarrow\rangle_{p}|\Delta \cdot \downarrow\rangle_{n}\left|{ }_{s} e^{e^{:} \cdot \nabla}\right\rangle_{y} .
$$

Here there are two descendants, with genuine uncertainty that cannot be removed even if they are informed about the quantum state. That is because their perception cannot distinguish whether they are in a rotated building or not. The situation is equivalent to Vaidman's [1998, 2014] sleeping pill thought experiment where the subject's entire body is split between two distant internally identical rooms. 
Recall the incoherence problem. We concede that there can be no uncertainty about the future in the MWI. The future-directed question: What is the probability of obtaining a particular result in a quantum measurement that will be performed? makes no sense. Our discussion of absent self-location uncertainty goes a step further. It illustrates that in some cases the pastdirected question: What is the probability that a particular result was obtained in a quantum measurement that has already been performed? also makes no sense. However, our discussion of tainted and clean self-location uncertainty shows that the past-directed question can make sense. And clean self-location uncertainty cannot be removed by complete knowledge of the physical state of the universe. Both tainted and clean self-location uncertainty can therefore be used as the basis for understanding probability in the MWI.

Wallace [2006: 4.2] has objected to this approach by stating that "in the bulk of experiments which we perform to test quantum mechanics the gap between our conducting the experiment and observing the result is too short to allow us time to be uncertain." Wallace concludes that "the Vaidman probabilities are somewhat contrived entities to use as the foundation of our epistemology of quantum mechanics."

Our response is that self-location uncertainty (and hence the applicability of the probability postulate) is ubiquitous. For example, just reading about the setup of a performed experiment usually puts one in a position of tainted self-location uncertainty. Before reading the results section one can apply the probability postulate to calculate the probability that one is in a world with a given set of results. ${ }^{1}$

Clean self-location uncertainty is indeed rare. But it is useful for showing why self-location probabilities should correspond to the Born rule and not some other rule. Indeed in the next section we will show that an agent in a position of clean self-location uncertainty can use her knowledge of the quantum state to reason her way towards the probability postulate.

\section{Proof of the Born rule}

A strong universal principle of physics is symmetry: if the physical situation has a particular symmetry, whatever will happen should respect this symmetry. In a deterministic single-world theory the principle has a simple manifestation: If there is no difference in the physical description of two places, and the same kind of measurements are performed in these places, there should be no difference in outcomes. If our theory is not deterministic, identical physical situations do not lead to identical outcomes. The manifestation of the principle is that the probabilities of the outcomes in the two places have to be the same. By applying this principle to collapse theories we can show that experiments exhibiting a certain symmetry comply with the Born rule.

The symmetry argument can also be applied in the (deterministic) many-worlds theory. When the experiment respects a symmetry, it will lead to a symmetry between descendants corresponding to the measurement outcomes. For such symmetric situations we can show that the probability of self-location complies with the probability postulate.

To generalise the proof beyond symmetric situations, we appeal to another physical principle, local supervenience: whatever happens in region $A$ depends only on the quantum description of this region and its immediate vicinity. ${ }^{2}$ The locality of all known interactions in nature is a strong argument in favor of local supervenience. However, if collapse is a physically real process then local supervenience is false since a measurement on a remote particle entangled with the

\footnotetext{
${ }^{1}$ We also agree with Tappenden [2011] that even if one is performing an experiment and knows that there will be no time for uncertainty, merely considering the predicament of a hypothetical post-measurement, pre-observation subject will be enough to guide the reasoning of a punter who believes the MWI.

${ }^{2}$ Vaidman [2012: p306] appeals to such a principle in a similar context. Later, Sebens and Carroll [2014, 2016] appealed to a variant, which we discuss in section 5 .
} 
particle in region $A$, changes the physical description in $A$. But we can still prove the Born rule for collapse theories provided that we replace the local supervenience principle with the relativistic axiom of no superluminal signalling. We start with this proof. Then we turn to the probability postulate in the MWI.

Note that although our application of the symmetry principle resembles the proof of the Born rule by Deutsch [1999] which was extended by Wallace [2012], the argument is different and it does not appeal to decision-theoretic principles.

\subsection{Proof of the Born rule in collapse theories}

\section{Step one: a particle in a symmetric superposition.}

Consider a particle in a superposition of three very far apart well localised wave packets centered at positions labeled $A, B$, and $C$. These three positions lie on the circumference of a perfect circle such that the particle has three-fold rotational symmetry. That is, the system appears the same when it is rotated by one third of a full turn about the circle's center. Three identical detectors, distributed over those same positions, which also exhibit three-fold rotational symmetry, simultaneously measure the presence of the particle.

The quantum mechanical description is as follows. The initial state of the particle is:

$$
\frac{1}{\sqrt{3}}(|a\rangle+|b\rangle+|c\rangle)=\frac{1}{\sqrt{3}}\left(|1\rangle_{\mathrm{a}}|0\rangle_{\mathrm{b}}|0\rangle_{\mathrm{c}}+|0\rangle_{\mathrm{a}}|1\rangle_{\mathrm{b}}|0\rangle_{\mathrm{c}}+|0\rangle_{\mathrm{a}}|0\rangle_{\mathrm{b}}|1\rangle_{\mathrm{c}}\right)
$$

where the right-hand-side is given in Fock representation, to help clarify the role that locality will play in our argument. The interaction process is described by

$$
\begin{aligned}
& \left.\frac{1}{\sqrt{3}}\left(|1\rangle_{\mathrm{a}}|0\rangle_{\mathrm{b}}|0\rangle_{\mathrm{c}}+|0\rangle_{\mathrm{a}}|1\rangle_{\mathrm{b}}|0\rangle_{\mathrm{c}}+|0\rangle_{\mathrm{a}}|0\rangle_{\mathrm{b}}|1\rangle_{\mathrm{c}}\right)|R\rangle_{\mathrm{A}}|R\rangle_{\mathrm{B}}|R\rangle_{\mathrm{C}}\right) \rightarrow \\
\rightarrow & \left.\frac{1}{\sqrt{3}}\left(|1\rangle_{\mathrm{a}}|0\rangle_{\mathrm{b}}|0\rangle_{\mathrm{c}}|\checkmark\rangle_{\mathrm{A}}|R\rangle_{\mathrm{B}}|R\rangle_{\mathrm{C}}+|0\rangle_{\mathrm{a}}|1\rangle_{\mathrm{b}}|0\rangle_{\mathrm{c}}|R\rangle_{\mathrm{A}}|\checkmark\rangle_{\mathrm{B}}|R\rangle_{\mathrm{C}}+|0\rangle_{\mathrm{a}}|0\rangle_{\mathrm{b}}|1\rangle_{\mathrm{c}}|R\rangle_{\mathrm{A}}|R\rangle_{\mathrm{B}}|\checkmark\rangle(6)\right)\right) \\
\rightarrow & \begin{cases}|1\rangle_{\mathrm{a}}|0\rangle_{\mathrm{b}}|0\rangle_{\mathrm{c}}|\checkmark\rangle_{\mathrm{A}}|R\rangle_{\mathrm{B}}|R\rangle_{\mathrm{C}} & \operatorname{prob}_{A} \\
|0\rangle_{\mathrm{a}}|1\rangle_{\mathrm{b}}|0\rangle_{\mathrm{c}}|R\rangle_{\mathrm{A}}|\checkmark\rangle_{\mathrm{B}}|R\rangle_{\mathrm{C}} & \operatorname{prob}_{B} \\
|0\rangle_{\mathrm{a}}|0\rangle_{\mathrm{b}}|1\rangle_{\mathrm{c}}|R\rangle_{\mathrm{A}}|R\rangle_{\mathrm{B}}|\checkmark\rangle_{\mathrm{C}} & \operatorname{prob}_{C}\end{cases}
\end{aligned}
$$

where $|R\rangle_{\mathrm{A}},|\checkmark\rangle_{\mathrm{A}}$ signify the ready and clicked states of the detector in $A$, etc. The symmetry between $A, B$ and $C$ is explicit before measurement interaction (5). It is still explicit during the measurement unitary evolution (6). The symmetry principle then also requires that it will be manifested in the mixture (7) after the measurement. Therefore, $\operatorname{prob}_{A}=\operatorname{prob}_{B}=\operatorname{prob}_{C}=\frac{1}{3}$.

This result is easily generalized from a three to an $N$ detector setup. This proves the Born rule for the special case of symmetric superpositions with equal coefficients.

\section{Step two: a particle described by a quantum state with local amplitude $\frac{1}{\sqrt{N}}$.}

Even if the measurement testing the presence of the particle is performed only in one place, the collapse happens simultaneously in all three places, breaking local supervenience. This feature of collapse makes many physicists doubt that it is a real physical process, but even among physicists who are ready to accept collapse, there is a consensus that it cannot lead to superluminal signaling. We now show that by assuming the impossibility of superluminal signalling (rather than local supervenience) we may generalize the proof.

If an action in a remote location can change the probability of finding the particle in $A$, we can send superluminal signals from this location to region $A$. Let us describe a concrete procedure. 
i). We prepare an ensemble with a large number $K$ of particles all prepared in the same state, say (4).

ii). We agree in advance that at time $t$, the observer in $A$ makes measurements testing the presence of particles in $A$.

iii). To send bit 0 to region $A$ from regions $B$ and $C$ we do nothing. The probability of finding every particle in $A$ is $\frac{1}{3}$ so the expectation value of the number of particles observed in $A$ will be $\left\langle K_{A}\right\rangle=\frac{K}{3}$.

iv). To send bit 1 to region $A$ from regions $B$ and $C$ we arrange unitary evolution of the wave packets $|b\rangle$ and $|c\rangle$ which makes them interfere into a single wave packet $|d\rangle$. The evolution of the quantum state of the particle due to this operation is:

$$
\frac{1}{\sqrt{3}}(|a\rangle+|b\rangle+|c\rangle) \rightarrow \frac{1}{\sqrt{3}}|a\rangle+\sqrt{\frac{2}{3}}|d\rangle .
$$

There is no symmetry in this state, so we should not expect that the observer in $A$ will find $\left\langle K_{A}\right\rangle=\frac{K}{3}$. For example, let us naively assume that if the wavefunction of the particle has identical shapes in different regions, then the probability of finding the particle in each region is the same, independently of the amplitudes in each region. In that case, since the wave packets at $A$ and $D$ have identical shapes, we expect that the observer in $A$ will find $\left\langle K_{A}\right\rangle=\frac{K}{2}$ particles. For any finite change of probability in $A$ due to actions in remote locations $(B$ and/or $C$ ) there is large enough $K$ such that the change will be observable. Indeed, the deviation in expectation value of the number of particles in $A$ is linear in $K$ while the uncertainty is proportional to $\sqrt{K}$, so violating the no signalling principle is easily achievable.

The shape of the localized wave packet in $A$ also cannot influence the probability without violating the no signalling principle. We can instantaneously change its phase, squeeze it, rotate it, change it locally in any way except for changing the absolute value of the total amplitude. This cannot change the probability to find the particle. If it does, the probability to find it elsewhere should be changed too (the total probability is 1), i.e. it is a superluminal signal from $A$ to another region.

So, we have come to the conclusion that to keep quantum mechanics consistent with relativity, the probability of finding a particle in a region $A$ has to be $\frac{1}{3}$ for every state which can be obtained from (4) by a change in the wavefunction outside region $A$ under the constraint that the light signal will not reach $A$ before time $t$.

In generalising the proof from a particle in a superposition of three locations on a circle to an arbitrary number $N$ locations on a circle, we will have difficulty interfering all parts but the part at $A$, before a subluminal signal about our actions could reach $A$. But we are not limited to doing this in one step. We can move the wavepackets, except the one in $A$ a finite distance before the signal reaches $A$. This will create a modified wavefunction which must still have probability $\frac{1}{N}$ to be found in $A$. We can move the part of the wavefunction of the modified configuration again, creating another state with probability $\frac{1}{N}$ to be found in $A$. We can therefore modify it in an arbitrary way while keeping amplitude $\frac{1}{\sqrt{N}}$ in $A$ and still claim that the probability to be found in $A$ is $\frac{1}{N}$.

Step three: a particle described by a quantum state with local amplitude $\sqrt{\frac{M}{N}}$.

Building on the previous step, it is easy to prove the Born rule for any rational coefficient. Consider the following wavefunction of the particle:

$$
\sqrt{\frac{M}{N}}|a\rangle+\sqrt{\frac{1}{N}} \sum_{i=1}^{N-M}\left|s_{i}\right\rangle .
$$


From the previous step we know that the probability to find the particle in each out of $N-M$ sites $S_{i}$ is $\frac{1}{N}$. Since the total probability to find it somewhere is 1 , the probability to find it in $A$ is $\frac{M}{N}$. To complete the proof we can apply the no superluminal signaling principle again. We can, in several steps, change the wavefunction outside region $A$ in an arbitrary way without a signal reaching the location $A$. Therefore, any wavefunction with the first term as in (9) must have probability $\frac{M}{N}$ to be found in $A$.

\section{Step four: proof of the Born rule for irrational coefficients.}

What if the amplitude is a square root of an irrational number? It seems clear that due to the continuity of everything in physics it should be just the absolute square of the amplitude again, otherwise there will be discontinuity as we can find a rational number arbitrarily close to any irrational number. If the continuity argument does not satisfy, Deutsch [1999] considered the case of irrational coefficients in detail from another angle.

Thus, we have established a general result: If the amplitude of the wavefunction in region $A$ is $\alpha$, the probability to find the particle there is $|\alpha|^{2}$. Of course, from our proof for one location, it follows for all locations: If the wavefunction is $\sum_{i} \alpha_{i}\left|A_{i}\right\rangle$ then the probability to find the particle in region $A_{i}$ is $\left|\alpha_{i}\right|^{2}$. This is the proof of the Born rule. This result followed from assuming symmetry and the requirement of compliance with relativity theory.

\section{Step five: proof of the Born rule for local quantum measurements.}

The proof above considers a quantum state of a particle placed in different locations, and it is not applicable directly to a quantum state of a local system like a spin. But our proof is a consistency check: Born rule violation is inconsistent with relativity theory. Born rule violation for a local measurement of a spin will also lead to inconsistency. Quantum theory considers various legitimate methods of measurements. In particular, we can arrange a unitary swap mechanism between the spin state and the spatial state of the particle:

$$
(\alpha|\uparrow\rangle+\beta|\downarrow\rangle)|\operatorname{ready}\rangle \rightarrow|\uparrow\rangle(\alpha|a\rangle+\beta|b\rangle),
$$

After this procedure we make a measurement of where the particle is. We have proved that this final measurement should comply with the Born rule, and the consistency of quantum theory tells us that direct local measurements of the spin should follow the Born rule too.

We have considered pure quantum states, but the consistency argument for a process with an additional interaction which creates entanglement extends the proof to measurements on systems described by mixed states.

We have therefore shown that symmetry and the principle of no superluminal signaling ensures Born rule probability assignments for the outcomes of quantum measurements.

\subsection{Proof of the probability postulate in the MWI}

In the MWI there is no physical process in addition to the relativistic generalisation of the Schrödinger equation. There is a principle which connects the wavefunction of the universe to us, to our experience. One's experience supervenes on the wavefunction of one's world, which is one of the possible collapsed wavefunctions of the collapse theory. This principle replaces the collapse principle of the standard approach. And there is a second principle, which we will now try to justify, our probability postulate.

Let us start again with a particle in a symmetric state (4). Three observers, Alex, Bob, and Charlie, are located at $A, B$, and $C$, respectively. To keep exact symmetry we might consider them as identical twins, but we see no reason why their personality should influence the outcomes of the experiments. We consider a sleeping pill situation for each observer. Alex sleeps in room 
$R$ (ready). If his device finds the particle (in $A$ ), he is moved to a nearby internally identical room $\checkmark$ (found). Given the MWI, Alex branches into a descendant who is still in room $R$ and a descendant who is placed in the $\checkmark$ room. The same situation is set up for Bob and Charlie in their distant locations.

We may now consider the perspective of one of Alex's descendants. What probability should he assign to self-locating in his $\checkmark$ room? Alex knows that if he is in his $\checkmark$ room then in his world, Bob and Charlie are in their $R$ rooms. Therefore, there are three exclusive possibilities (Alex $\checkmark$, Bob $\checkmark$, Charlie $\checkmark$ ), and the sum of their probabilities must equal one. Alex also understands the symmetry of the physical situation. It ensures that Alex, Bob, and Charlie should each assign the same self-locating probability to finding themselves in a $\checkmark$ room. Therefore, the probability that Alex should assign to finding himself in his $\checkmark$ room is $\frac{1}{3}$. Clearly, the argument is the same if we have more than three observers distributed symmetrically. So, at least for such symmetric situations, the probability postulate follows.

The quantum description of this situation reflects the symmetry of the problem, apart from inessential differences between Alex, Bob, and Charlie. We are interested in the local description at location $A$ after the measurement and after moving the observers. The complete local quantum description of $A$ is given by the reduced density matrix:

$$
\frac{1}{3}|1\rangle_{\mathrm{a}}|\checkmark\rangle_{\mathrm{A}}|\checkmark\rangle_{\mathrm{Al}}\left\langle\left.\checkmark\right|_{\mathrm{Al}}\left\langle\left.\checkmark\right|_{\mathrm{A}}\left\langle\left. 1\right|_{\mathrm{a}}+\frac{2}{3} \mid 0\right\rangle_{\mathrm{a}} \mid R\right\rangle_{\mathrm{A}} \mid R\right\rangle_{\mathrm{Al}}\left\langleR | _ { \mathrm { Al } } \left\langleR | _ { \mathrm { A } } \left\langle\left. 0\right|_{\mathrm{a}}\right.\right.\right.
$$

where $|R\rangle_{\mathrm{A}}$ and $|\checkmark\rangle_{\mathrm{A}}$ signify ready and found states of the detector at $A$, and $|R\rangle_{\mathrm{Al}}$ and $|\checkmark\rangle_{\mathrm{Al}}$ signify states of Alex in different rooms.

The next step of the argument considers a situation which differs from the previous situation only outside region $A$. Instead of detecting the particle in $B$ and $C$, the wave packets in $B$ and $C$ are redirected to a beamsplitter and after interference reach a detector in location $D$ where a sleeping Daniel (an observer similar to Alex, Bob, and Charlie) is moved to room $\checkmark$ if the detector finds the particle. The quantum description is not symmetric anymore, but the local description in $A$ remains the same (11). Local supervenience tells us that Alex should assign probabilities for self-locating in the rooms in the same way as in the symmetric case.

The local reduced density matrix in $A$ remains the same as in the first case. Consequently, Alex was not informed about the redirection of the particle wave toward Daniel's detector. So, of course he will have the same expectations about self-location: room $\checkmark$ or room $R$. The question is: Does the redirection of the particle make his probability assignment incorrect?

Recall the local supervenience principle: whatever happens in region $A$ depends on the quantum description of this region and its immediate vicinity. This principle is justified by the locality of the physics of the MWI, where the physics is understood as the wavefunction evolving in accord with the relativistic generalisation of the Schrödinger equation. ${ }^{3}$ Since Alex's probability assignment concerns what happens in region $A$, it must depend solely on the quantum description of this region. Since that description does not change, neither does the correctness of Alex's probability assignment. It is therefore the locality of the physics that explains why individuals should trust the probability postulate even in situations without symmetry.

Our argument assumes that probability assignments in the MWI are physically testable. It has been claimed, however, that the probabilistic predictions in MWI suffer a problem of confirmation [Greaves and Myrvold, 2010]. If Alex's probability assignment cannot be confirmed by observing physical events in $A$, then there is no reason to suppose that local supervenience, grounded in physics, applies to such assignments. But we do have a confirmation procedure. Operationally, the observer performs a large number of measurements and compares the observed statistics with the Born rule. It tests for the probability rule regarding the observer's self-location.

\footnotetext{
${ }^{3}$ The locality of the physics of the MWI is recently discussed in Wallace [2012: sec. 8.5.1] and Vaidman [2016].
} 
One can only observe one's own world, and so confirmation of the probability rule concerns whether our current records correspond to Born rule statistics. Our empirical evidence is that it does.

This confirmation procedure tests the probability rules of all interpretations of quantum mechanics. ${ }^{4}$ In the collapse theory, the procedure tests for an objective random process manifested in the records of the (only) observer in region $A$. Like any finite test of a probabilistic theory, it might fail. The failure can occur both for the MWI and for a collapse theory. Moreover, if we consider test runs with, say, one thousand experiments, we should expect that some of them will fail when the thousand-experiment runs are repeated, say, a googol times. It is the absence of failures in this multiple run of tests that will provide a refutation of the theory. Some objections to the possibility of confirmation in the MWI are considered in the next section.

The remaining steps of the proof can be done as in the case of the collapse theory. The key difference between the collapse and the MWI proofs is that we need no additional relativistic argument in the MWI proof since nonlocal collapse is not present in the MWI. Local relativistic causality is included in the relativistic generalisation of the Schrödinger equation.

\subsection{Discussion of the probability postulate}

For some of Alex's descendants the Born rule seems correct, for others, it seems not to be. In the case of collapse, we have one descendant and we agreed to neglect the small probability cases when he does not observe Born rule statistics. But in the MWI we are certain that there are descendants who don't observe Born rule statistics. Apparently, it is considerations like these that cause Albert [2015: p162] to question whether the MWI is susceptible of confirmation. He asks: Why (for example) should it come as a surprise, on a picture like this, to see what we would ordinarily consider a low-probability string of experimental results? Why should such a result cast any doubt on the truth of this theory (as it does, in fact, cast doubt on quantum mechanics)?

We think objections like these are misguided: the objector is trying to determine what probability rule is confirmed by the MWI ontology by considering specific worlds. Thus, the objector considers maverick worlds (worlds that appear to defy the Born rule), and then concludes that the Born rule is not confirmed. The objections do not adequately distinguish between confirmation and deduction of a probability rule. We confirm a probability rule by inferring it from observations. We deduce a probability rule by proving it from our theory. The former requires considering only our records since those are the only records we can observe. The latter requires considering everything that the theory encompasses and in the MWI that requires taking all worlds and all descendant's records into consideration.

Regarding confirmation, our observations are confined to our records and it is clear that those records confirm the probability postulate. Regarding deduction, we have already offered our proof. However, some have suggested that the MWI may in fact entail alternative incorrect probability rules. The main example is the naive counting measure. To illustrate, recall our symmetric case of a particle moving to $A, B$, and $C$. Here naive counting correctly deduces the Born rule by the majority vote: in the majority of worlds the frequency of result $A$ is close to $\frac{1}{3}$. But, if we consider the ensemble of measurements with a particle redirected to $D$, in the majority of worlds the frequency of result $A$ is close to $\frac{1}{2}$. Here the naive counting measure diverges from the Born rule. This, however, should not be valid, because a naive majority vote with unequal amplitude worlds is illegitimate. In the equal case, symmetry is what makes majority vote

\footnotetext{
${ }^{4}$ We therefore disagree with some MWI advocates who either think that the MWI requires an unorthodox theory of confirmation, or exhibits some advantage over collapse theories regarding the issue of probability [cf. Deutsch, 2016].
} 
legitimate. But when this symmetry is lost, there is no a priori reason to treat all descendants on a par. Doing so would be like treating all American states on a par, if one were blindfolded, dropped at a random point in America, and asked to assign self-location probabilities to each state.

In the MWI there is a well-defined alternative strategy that weights descendants in proportion to their absolute squared amplitudes (or equivalently, their measures of existence). It is attractive because it ensures consistency with the situations in which majority vote is legitimate: every $D$-world can be split into two equal amplitude worlds with amplitude equal to the $A$ world. Then, all worlds have the same amplitude and majority vote tells us that the probability for Daniel to self-locate in some $D$ world is $\frac{1}{3}+\frac{1}{3}=\frac{2}{3} \cdot 5$

Hemmo and Pitowsky [2007] ask why our frequencies would be any more indicative of the true theory, than the frequencies observed by experimenters in maverick worlds? Indeed, perhaps such experimenters have concocted their own physical theory, and their own probability rule, and are exploiting symmetries in their theories, to provide proofs of the probability rule that their frequencies support.

In response, such experimenters can reach the Born rule by considering explanations of natural phenomena. Consider the explanation of the sky being blue. The sun sends to Earth an enormous number of photons of different colors. The frequency of blue light corresponds to the natural resonant frequency of the bound electrons in atmospheric molecules. Consequently, the molecules strongly scatter the blue photons. The frequency of red is far from the resonance frequency of molecules, so they are scattered much less. Quantum mechanically, each photon actually ends up in a superposition of scattered and undisturbed states. The difference is that blue light scatters with much higher amplitude than red light. Eventually the light reaches our retina and branching occurs. To explain why we see blue sky we need to appeal to the high amplitude of blue photon scattering and so to the Born rule which connects the probability of absorption with this amplitude.

Thus, agents in maverick spin-measurement worlds, despite their maverick statistics in the lab, will eventually discover quantum theory (and the probability postulate) anyway, by focusing on explanations of natural phenomena, like the sky being blue.

It might seem like this pushes the problem to red-sky maverick worlds. But now there are two responses: (i) once we start talking about worlds with different natural processes, we begin talking about worlds that may not support life and therefore measurement outcomes; and (ii) even if such worlds do support life, we find it very implausible that it would be possible to construct a reasonable theory of natural phenomena in such worlds.

Note that this argument applies equally well to collapse theories. Just as the MWI predicts maverick worlds, collapse theories predict maverick possibilities. Consequently, we can ask Albert's question: Why should it come as a surprise, on a collapse theory, to see what we would ordinarily consider a low-probability string of experimental results? After all, such a string is logically consistent with a collapse theory. The answer is the same as in the MWI: there is no reason for why one should take such a string to refute collapse theories if a much broader set of data (including e.g. the reason for the sky's colour) supports the Born rule.

This ends our defence of the self-location uncertainty account, as we understand it. In the remainder of the paper we compare our account to Sebens and Carroll's [forthcoming], and we show that our account does not fall prey to Kent's [2015] critique of their account.

\footnotetext{
${ }^{5}$ Moreover, majority voting in realistic cases requires there to be a well-defined number of parallel worlds (Wallace [2012, sec. 3.11]). The measure of existence of a world avoids this difficulty.
} 


\section{Critique of Sebens and Carroll's proof}

Kent [2015] has questioned whether it makes sense to speak of self-location uncertainty in the MWI. In particular, he asks "whether it is meaningful to say that observers are uncertain about their self-location in the universal wavefunction, and can meaningfully assign probabilities to the events that they are located in one branch or another" [2015: p212]. However, Kent's critique is given in the context of considering the recent account offered by Sebens and Carroll [forthcoming]. ${ }^{6}$ We think Kent's concerns about their approach are well-founded, but only because of weaknesses specific to their approach.

Kent analyses Sebens and Carroll's [2014: 161, eqn. 10.8] description of the quantum state of a spin- $\frac{1}{2}$ particle, apparatus $A$, observer $O$, and environment $\omega$, prior to the observer recording the outcome of the measurement:

$$
\frac{1}{\sqrt{2}}\left(\left|O_{0}\right\rangle|\uparrow\rangle\left|A_{\uparrow}\right\rangle\left|\omega_{\uparrow}\right\rangle+\left|O_{0}\right\rangle|\downarrow\rangle\left|A_{\downarrow}\right\rangle\left|\omega_{\downarrow}\right\rangle\right) .
$$

Kent takes issue with Sebens and Carroll's claim - which is crucial to their account - that "The observer is still described by a unique state $\left|O_{0}\right\rangle$, but there are two copies, one in each branch. Such an observer is in a state of self-location uncertainty" [Carroll and Sebens, 2014: p161]. Kent [2015: p213-4] responds:

These are bold, and prima facie peculiar, asssertions, which seem to need careful justficiation and explanation. [...] Why can we not say [...] that it represents one observer? On this latter view, our sole observer has not interacted with the entangled particle-apparatus-environment state, and has no uncertainty about this state, or their "branch location", or anything else.

We agree with Kent. In fact, Sebens and Carroll's equation (12) is analogous to our equation (1) and represents a case of absent self-location uncertainty. In this situation there is only one observer and so there cannot be a question of her self-location. Indeed there are two worlds in this situation but she is located in both. All of the equations that are central to Sebens and Carroll's proof of the Born rule describe cases of absent self-location uncertainty. Consequently, their proof cannot work, since it concerns cases where probability is non-existent. Our proof avoids Kent's criticism since it concerns cases involving clean self-location uncertainty.

One might wonder whether their proof could be amended so that it instead involves cases of tainted or clean self-location uncertainty. We will show that it cannot.

The central principle in their proof - the epistemic separability principle (ESP) - is as follows [forthcoming: p16]:

"ESP-gist The credence one should assign to being any one of several observers having identical experiences is independent of the state of the environment."

Our concern is not with the principle itself, but with the fact that it is employed in situations in which several observers do not exist. For the purpose of their proof, the informal "gist' is given a formal statement:

"ESP-QM Suppose that an experiment has just measured observable $\hat{O}$ of system $S$ and registered some eigenvalue $O_{i}$ on each branch of the wave-function. The probability that agent $A$ ought to assign to the detector $D$ having registered $O_{i}$ when the universal wave-function is $\psi, P\left(O_{i} \mid \psi\right)$, only depends on the reduced density matrix of $A$ and $D, \hat{\rho}_{A D}$ :

\footnotetext{
${ }^{6}$ See also [Carroll and Sebens 2014].
} 


$$
P\left(O_{i} \mid \psi\right)=P\left(O_{i} \mid \hat{\rho}_{A D}\right) .
$$

This principle tells us that when observers assign probabilities to recorded outcomes of measurements that have already occurred, these probabilities should depend on the Agent+Detector state, $\hat{\rho}_{A D}$ (not on other features of the universal wave-function)."

We grant them this principle too, provided that probability has meaning in the situation under consideration. This requires either clean or tainted cases of self-location uncertainty. But appeal to such cases will invalidate their proof.

Consider the first step of their proof of the Born rule for the equal amplitude case [forthcoming: p19].

"Alice measures the z-spin of a single particle in the x-spin up state. One display $(D 1)$ will show the result of the experiment. If the spin is up, a second display $(D 2)$ will show $\varnothing$. If it is down, a $\diamond$ will appear on the second display."

We now have a crucial stipulation:

"Alice is not immediately affected by the result; in particular, she is for a time unaware of the experiment's outcome. That is, we assume that Alice is in the same physical state on both branches of the wave-function $\left(|R\rangle_{A}\right.$ in (15) [below])."

However, if Alice has not been affected, then she is in a position of absent self-location uncertainty, since there is only one of her. Consequently, for her there is no meaning to the question "which world am I in?", she is in both worlds. But let us continue with the proof.

"The wave-function of Alice, the detectors, the particle, and the environment (the rest of the world) evolves from

$$
\left|\psi_{0}\right\rangle=\left|R_{0}\right\rangle_{A}|R\rangle_{D 1}|R\rangle_{D 2}\left|\uparrow_{x}\right\rangle\left|E_{R}\right\rangle
$$

to

$$
\left|\psi_{1}\right\rangle=\frac{1}{\sqrt{2}}|R\rangle_{A}|\uparrow\rangle_{D 1}|\nabla\rangle_{D 2}\left|\uparrow_{z}\right\rangle\left|E_{\uparrow \odot}\right\rangle+\frac{1}{\sqrt{2}}|R\rangle_{A}|\downarrow\rangle_{D 1}|\diamond\rangle_{D 2}\left|\downarrow_{z}\right\rangle \mid E_{\downarrow \diamond\rangle} .
$$

To use ESP-QM to demonstrate that $P\left(\uparrow \mid \psi_{1}\right)=P\left(\downarrow \mid \psi_{1}\right)=\frac{1}{2}$, we will need to also consider an alternate scenario where the computer (part of the environment) is programmed differently so that $\odot$ displays if down is measured and $\diamond$ displays if up. Then the post-measurement, pre-observation wave-function would be:

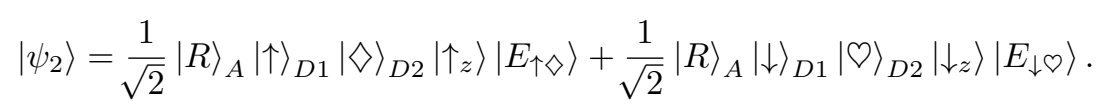

Step 1: Focus first on Alice and D1. The (Alice + Detector 1) reduced density matrices for $\psi_{1}$ and $\psi_{2}$ are the same, 


$$
\hat{\rho}_{A D 1}\left(\psi_{1}\right)=\hat{\rho}_{A D 1}\left(\psi_{2}\right)=\frac{1}{2}|R\rangle_{A}|\uparrow\rangle_{D 1}\left\langle\left. R\right|_{A}\left\langle\left.\uparrow\right|_{D 1}+\frac{1}{2} \mid R\right\rangle_{A} \mid \downarrow\right\rangle_{D 1}\left\langleR | _ { A } \left\langle\left.\downarrow\right|_{D 1} .\right.\right.
$$

ESP-QM requires that the probabilities Alice assigns to the possible spin-results be the same in these two universes as they have the same (Observer + Detector) reduced density matrix,

$$
P\left(\downarrow \mid \psi_{1}\right)=P\left(\downarrow \mid \psi_{2}\right) .
$$

Step 2: If we ask what probability Alice should assign to the display being $\varnothing$, we need to consider the reduced density matrix generated by tracing over $D 1$, the spin of the particle, and the environment. $\psi_{1}$ and $\psi_{2}$ agree on $\hat{\rho}_{A D 2}$. By ESP-QM, the probabilities assigned to $\odot$ must be equal,

$$
P\left(\odot \mid \psi_{1}\right)=P\left(\odot \mid \psi_{2}\right) . "
$$

We now consider whether these two steps can be reconstructed by replacing these equations, which describe situations of absent self-location uncertainty, with equations that describe situations of tainted or clean self-location uncertainty. We begin by replacing (15) and (16) with corresponding equations in which Alice is entangled with the particle's spin:

$$
\begin{aligned}
& \left|\psi_{1}^{\prime}\right\rangle=\frac{1}{\sqrt{2}}\left|R_{\uparrow}\right\rangle_{A}|\uparrow\rangle_{D 1}|\circlearrowleft\rangle_{D 2}\left|\uparrow_{z}\right\rangle\left|E_{\uparrow \odot}\right\rangle+\frac{1}{\sqrt{2}}\left|R_{\downarrow}\right\rangle_{A}|\downarrow\rangle_{D 1}|\diamond\rangle_{D 2}\left|\downarrow_{z}\right\rangle\left|E_{\downarrow \diamond\rangle}\right\rangle \\
& \left|\psi_{2}^{\prime}\right\rangle=\frac{1}{\sqrt{2}}\left|R_{\uparrow}\right\rangle_{A}|\uparrow\rangle_{D 1}|\diamond\rangle_{D 2}\left|\uparrow_{z}\right\rangle\left|E_{\uparrow \diamond}\right\rangle+\frac{1}{\sqrt{2}}\left|R_{\downarrow}\right\rangle_{A}|\downarrow\rangle_{D 1}|\oslash\rangle_{D 2}\left|\downarrow_{z}\right\rangle\left|E_{\downarrow}\right\rangle .
\end{aligned}
$$

Here $\left|R_{\uparrow}\right\rangle_{A}$ represents Alice in the ready (pre-observation) state, while also being entangled with the spin of the particle. It does not matter for our argument whether we treat these as cases of tainted, or cases of clean, self-location uncertainty.

Let us see if we can use ESP-QM to demonstrate that $P\left(\uparrow \mid \psi_{1}^{\prime}\right)=P\left(\downarrow \mid \psi_{1}^{\prime}\right)=\frac{1}{2}$. Step 1: Focus first on Alice and $D 1$. The (Alice + Detector 1) reduced density matrices for $\psi_{1}^{\prime}$ and $\psi_{2}^{\prime}$ are the same,

$$
\hat{\rho}_{A D 1}\left(\psi_{1}^{\prime}\right)=\hat{\rho}_{A D 1}\left(\psi_{2}^{\prime}\right)=\frac{1}{2}\left|R_{\uparrow}\right\rangle_{A}|\uparrow\rangle_{D 1}\left\langle\left. R_{\uparrow}\right|_{A}\left\langle\left.\uparrow\right|_{D 1}+\frac{1}{2} \mid R_{\downarrow}\right\rangle_{A} \mid \downarrow\right\rangle_{D 1}\left\langleR _ { \downarrow } | _ { A } \left\langle\left.\downarrow\right|_{D 1} .\right.\right.
$$

ESP-QM requires that the probabilities Alice assigns to the possible spin-results be the same in these two universes as they have the same (Observer + Detector) reduced density matrix,

$$
P\left(\downarrow \mid \psi_{1}^{\prime}\right)=P\left(\downarrow \mid \psi_{2}^{\prime}\right) .
$$

So far, so good. But things go inevitably wrong at Step 2: If we ask what probability Alice should assign to the display being $\varnothing$, we need to consider the reduced density matrix generated by tracing over $D 1$, the spin of the particle, and the environment. However, it is not the case that $\psi_{1}^{\prime}$ and $\psi_{2}^{\prime}$ agree on $\hat{\rho}_{A D 2}$ :

$$
\begin{aligned}
& \hat{\rho}_{A D 2}\left(\psi_{1}^{\prime}\right)=\frac{1}{2}\left|R_{\uparrow}\right\rangle_{A}|\nabla\rangle_{D 2}\left\langle\left. R_{\uparrow}\right|_{A}\left\langle\left.\nabla\right|_{D 2}+\frac{1}{2} \mid R_{\downarrow}\right\rangle_{A} \mid \nabla\right\rangle_{D 2}\left\langleR _ { \downarrow } | _ { A } \left\langle\left.\diamond\right|_{D 2}\right.\right. \\
& \hat{\rho}_{A D 2}\left(\psi_{2}^{\prime}\right)=\frac{1}{2}\left|R_{\uparrow}\right\rangle_{A}|\nabla\rangle_{D 2}\left\langle\left. R_{\uparrow}\right|_{A}\left\langle\left.\diamond\right|_{D 2}+\frac{1}{2} \mid R_{\downarrow}\right\rangle_{A} \mid \nabla\right\rangle_{D 2}\left\langleR _ { \downarrow } | _ { A } \left\langle\left.\nabla\right|_{D 2} .\right.\right.
\end{aligned}
$$


And since $\hat{\rho}_{A D 2}\left(\psi_{1}^{\prime}\right) \neq \hat{\rho}_{A D 2}\left(\psi_{2}^{\prime}\right)$, ESP-QM cannot be used to establish the desired conclusion

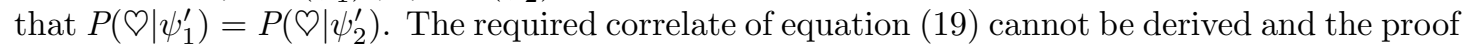
fails.

Our critique of Sebens and Carroll's proof is based on our section 3 analysis, which distinguished absent, tainted, and clean self-location uncertainty. Sebens and Carroll may respond to this analysis. Let us finish by considering two possible responses.

Contrary to our analysis, Sebens and Carroll [forthcoming: p12] argue that self-location uncertainty is always inevitable in any quantum measurement. For example, in all of our notebook cases, their analysis entails that you will enter a brief period of self-location uncertainty as you open the notebook. They say:

"For any realistic room-temperature experimental apparatus, the decoherence time is extremely short: less than $10^{-20}$ seconds. Even if a human observer looks at the quantum system directly, the state of the observer's eyeballs will decohere in a comparable time. In contrast, the time it takes a brain to process a thought is measured in tens of milliseconds. [Therefore] No matter what we do, real observers will find themselves in a situation of self-location uncertainty (after decoherence, before the measurement outcome has been registered)."

In response, it is far from obvious that self-location uncertainty obtains in this brief period. Consider the brief moment in which Alice's eyes are entangled with the display, but her cognitive processing system is not. If we break Alice's quantum state $(A)$ down into the state of her retina $(R)$ and the state of her cognitive processing system $(C)$, then in this period we have:

$$
\frac{1}{\sqrt{2}}\left(|“ \uparrow "\rangle_{D 1}|“ \uparrow "\rangle_{R}+|“ \downarrow "\rangle_{D 1}|“ \downarrow "\rangle_{R}\right)|\operatorname{Ready}\rangle_{C} .
$$

On a natural understanding of your "self", you are not your eyeballs, rather, you are your cognitive system. But if that is right, then there is still no meaningful question of self-location, even in the minuscule period that Sebens and Carroll refer to. That is, (26) describes a case of absent self-location uncertainty.

But to make our point we do not need to settle the difficult question of what constitutes the self. $^{7}$ Let us grant that Alice can be identified with both her cognitive system and her eyes such that there are two Alice's even when her eyes are the only part of her that have branched. In that brief moment it is not the case that $\psi_{1}^{\prime}$ and $\psi_{2}^{\prime}$ agree on $\hat{\rho}_{A D 2}$, and so the proof still fails.

This brings out an in-principle problem for any proof that makes use of both ESP-QM and self-location probability: the latter idea only makes sense when the former is inapplicable.

A second possible response to our argument takes issue with our discussion of absent selflocation uncertainty. Contrary to our analysis, Sebens and Carroll work under the assumption that "branching happens throughout the wavefunction whenever it happens anywhere" (p10). Consequently, "observers here on Earth could be (and almost surely are) branching all the time, without noticing it, due to quantum evolution of systems in the Andromeda Galaxy" (p11). Sebens and Carroll (p10 note 6) note that this picture "plays an important role" in allowing them to examine cases where the agent is in a physically identical state on each branch (e.g. the case of Alice). Our argument can be interpreted as showing that it plays an essential role.

\footnotetext{
${ }^{7}$ We do use some metaphysical assumptions about "the self". It must be well localized in space and time. We do not consider the self as a "history" in a sense which leads to a "divergent" instead of an overlapping picture [Saunders, 2010; Wilson 2012]. Sebens and Carroll use the overlapping picture although they note that their strategy "can be implemented on either picture" [sec. 2.1]. And we do not consider a self's "parting lines" as recently proposed by Tappenden [2017].
} 
Sebens and Carroll concede that this global branching picture is psychologically unintuitive (p11). But it also goes against the spirit of the many worlds interpretation, which involves removing as much nonlocality as possible. Thus, after removing the nonlocality of collapse, they reinsert a different kind of nonlocality. The new nonlocality involves branching events happening arbitrarily far away triggering branching events here on Earth. And this insertion has no apparent independent motivation. It just enables the proof to go through, rendering it ad hoc. On top of this, the global branching picture may even call into doubt the key epistemic separability principle, which states that the credence one should assign to being any one of several observers having identical experiences is independent of the state of the environment. This idea is meant to have intuitive appeal. But it is unclear why we should believe it if events arbitrarily far away can cause one to branch into multiple observers.

Stepping back from metaphysical speculations about alternative branching ontologies, what our technical analysis shows is that the proof fails because in the relevant scenario there is just one quantum description of the observer meaning that the observer has not branched. But if the observer has not branched then self-location probabilities cannot be introduced.

\section{Conclusion}

We have defended the MWI against the objection that it cannot explain why measurement outcomes correspond to the Born rule. We explained quantum probabilities in terms of selflocation probabilities and formulated a corresponding probability postulate for the MWI. We offered a proof of this postulate that appeals to ontological features of the MWI: symmetry and locality. Symmetry was used in a different way than in other approaches, and we showed that the symmetry under exchange argument by Sebens and Carroll does not work. Finally, we responded to objections, which focus on the possibility of confirmation and on the very notion of self-location uncertainty.

Do we provide unconditional proof of the probability postulate in the MWI from the formalism of quantum mechanics as Everett and others hoped possible? Deustch [1999] and Wallace [2012] relied on some decision-theoretic principles of rationality to deduce their Born rule analogue. We used a particular metaphysical approach that relied on physical principles. ${ }^{8}$ Similar principles allowed us to "prove" the Born rule in the framework of standard quantum mechanics. The status of our proofs therefore greatly depends on the status of these physical principles. We welcome further research into these principles. Either way, we have shown that probability can be consistently and naturally introduced into the framework of the MWI and that there is no special advantage that collapse theories have over the MWI regarding derivation of the Born rule. ${ }^{9}$

\section{$7 \quad$ References}

Albert, D. Z. 2015. After Physics. Harvard University Press.

Barrett, J. A. 2017. Typical worlds. Studies in History and Philosophy of Modern Physics $58,31-40$.

\footnotetext{
${ }^{8}$ This agrees with the recent conclusion of Barrett [2017] "that pure wave mechanics requires a number of significant auxiliary assumptions in order to make anything like the standard quantum predictions."

${ }^{9}$ The authors would like to thank David Albert, Justin Dressel, Meir Hemmo, and Charles Sebens, for helpful comments and discussion. This work has been supported in part by the Israel Science Foundation Grant No $1311 / 14$.
} 
Bohm, D. 1952. A suggested interpretation of the quantum theory in terms of "hidden" variables, I and II. Physical Review 85, 166.

Carroll, S.M. and 2014. Many Worlds, the Born Rule, and Self-location Uncertainty. In Daniele C. Struppa and Jeffrey M. Tollaksen (eds.), Quantum Theory: A Two-Time Success Story. Springer: 157-169.

Deutsch, D. 1999. Quantum theory of probability and decisions. Proceedings of the Royal Society of London. A 455, 3129.

Deutsch, D. 2016. The logic of experimental tests, particularly of Everettian quantum theory. Studies in History and Philosophy of Modern Physics 55, 24-33.

Everett, H. 1957. Relative state formulation of quantum mechanics. Reviews of Modern Physics 29: 454-462.

Ghirardi, G.C., A. Rimini, and T. Weber. 1986. Unified dynamics for microscopic and macroscopic systems. Physical Review D 34, 470.

Greaves, H. 2004. Understanding Deutsch's Probability in a Deterministic Multiverse. Studies in History and Philosophy of Modern Physics 35, 423-56.

Greaves, H. and Myrvold 2010. Everett and Evidence. in Many Worlds?, Saunders, S., Barrett, J., Kent, A., \& Wallace, D. eds. Oxford University Press.

Groisman, B., N. Hallakoun, and L. Vaidman. 2013. The measure of existence of a quantum world and the Sleeping Beauty Problem. Analysis 73, 695-706.

Hemmo and Pitowsky, 2007. Quantum probability and many worlds. Studies in History and Philosophy of Modern Physics 38,2: 333-350

Kent, A. 2015. Does it Make sense to speak of self-location uncertainty in the universal wave-function? Remarks on Sebens and Carroll. Foundations of Physics 45: 211-217.

Lewis, P. 2007. Uncertainty and probability for branching selves. Studies in History and Philosophy of Science Part B 38,1: 1-14.

Lewis, P. 2016. Quantum Ontology. Oxford University Press.

Pearle, P. 1976. Reduction of the state vector by a nonlinear Schroedinger equation. Physical Review. D 13, 857.

Saunders, S. 2010. Chance in the Everett Interpretation, in Many Worlds?, Saunders, S., Barrett, J., Kent, A., \& Wallace, D. eds. Oxford University Press.

Sebens, C.T. and Carroll, S.M. forthcoming. Self-location Uncertainty and the Origin of Probability in Everettian Quantum Mechanics. British Journal for the Philosophy of Science. 
doi: 10.1093/bjps/axw004.

Tappenden, P. 2011. Evidence and uncertainty in Everett's multiverse. British Journal for the Philosophy of Science 62, 99.

Tappenden, P. 2017. Objective probability and the mind-body relation. Studies in History and Philosophy of Science Part B: Studies in History and Philosophy of Modern Physics 57, 8.

Vaidman, L. 1998. On schizophrenic experiences of the neutron or why we should believe in the Many-Worlds interpretation of quantum theory. International Studies in the Philosophy of Science. 12, 245.

Vaidman, L. 2012. Probability in the Many-Worlds interpretation of quantum mechanics, in Probability in Physics, The Frontiers Collection, Y. Ben-Menahem and M. Hemmo, eds., p. 299 Springer-Verlag, Berlin Heidelberg.

Vaidman, L. 2014. Many-worlds interpretation of quantum mechanics. In E. Zalta (Ed.), The Stanford Encyclopedia of Philosophy. http://plato.stanford.edu/entries/qm-manyworlds/.

Vaidman, L. 2012. Probability in the Many-Worlds interpretation of quantum mechanics, in Probability in Physics, The Frontiers Collection, Y. Ben-Menahem and M. Hemmo, eds., p. 299 Springer-Verlag, Berlin Heidelberg.

Vaidman, L. 2016. Bell Inequality and Many-Worlds Interpretation, in Quantum Nonlocality and Reality, M. Bell and G. Shan, eds., Cambridge University Press.

Wallace, D. 2006. Epistemology quantized: circumstances in which we should come to believe in the Everett interpretation. British Journal for the Philosophy of Science, 57: 655-89.

Wallace, D. 2012. The Emergent Multiverse: Quantum Theory according to the Everett Interpretation. Oxford: Oxford University Press.

Wilson, A. 2013. Objective probability in Everettian quantum mechanics. British Journal for the Philosophy of Science 64, 709-737. doi:10.1093/bjps/axs022 